\title{
Research Paper: Changes in Effective Connectivity Network Patterns in Drug Abusers, Treated With Different Methods

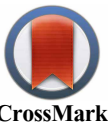

Arash Zare Sadeghi ${ }^{1,2}$, Amir Homayoun Jafari ${ }^{2 *}$, Mohammad Ali Oghabian ${ }^{1,2}$, Hamid Reza Salighehrad ${ }^{1}$, Seyed Amir Hossein Batouli ${ }^{2}$, Samira Raminfard $^{2,3}$, Hamed Ekhtiari ${ }^{2}$

1. Department of Medical Physics and Biomedical Engineering, School of Medicine, Tehran University of Medical Sciences, Tehran, Iran. 2. Department of Nouroimaging and Analysis, Imam Khomeini Hospital Complex, Tehran University of Medical Sciences, Tehran, Iran.

3. Department of Neurosciences and Addiction Studies, School of Advanced Technologies in Medicine, Tehran University of Medical Sciences, Tehran, Iran

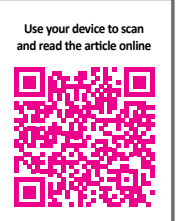

Citation: Zare Sadeghi, A., Jafari, A. H., Oghabian, M. A., Salighehrad, H. R., Batouli, S. A. M., Raminfard, S., et al. (2017). Changes in Effective Connectivity Network Patterns in Drug Abusers, Treated With Different Methods. Journal of Basic and

Clinical Neuroscience, 8(4), 285-298. https://doi.org/10.18869/NIRP.BCN.8.4.285

: https://doi.org/10.18869/NIRP.BCN.8.4.285

Article info:

Received: 19 Sep 2016

First Revision: 26 Oct. 2016

Accepted: 10 Feb. 2017
Key Words:

Dynamic causal modeling, Functional magnetic resonance imaging, Abstinent based therapy, Methadone maintenance therapy

\begin{abstract}
A B S T RA C T
Introduction: Various treatment methods for drug abusers will result in different success rates This is partly due to different neural assumptions and partly due to various rate of relapse in abusers because of different circumstances. Investigating the brain activation networks of treated subjects can reveal the hidden mechanisms of the therapeutic methods.

Methods: We studied three groups of subjects: heroin abusers treated with abstinent based therapy (ABT) method, heroin abusers treated with Methadone Maintenance Therapy (MMT) method, and a control group. They were all scanned with functional magnetic resonance imaging (fMRI), using a 6-block task, where each block consisted of the rest-craving-rest-neutral sequence. Using the dynamic causal modeling (DCM) algorithm, brain effective connectivity network (caused by the drug craving stimulation) was quantified for all groups. In this regard, 4 brain areas were selected for this analysis based on previous findings: ventromedial prefrontal cortex (VMPFC), dorsolateral prefrontal cortex (DLPFC), amygdala, and ventral striatum.
\end{abstract}

Results: Our results indicated that the control subjects did not show significant brain activations after craving stimulations, but the two other groups showed significant brain activations in all 4 regions. In addition, VMPFC showed higher activations in the ABT group compared to the MMT group. The effective connectivity network suggested that the control subjects did not have any direct input from drug-related cue indices, while the other two groups showed reactions to these cues. Also, VMPFC displayed an important role in ABT group. In encountering the craving pictures, MMT subjects manifest a very simple mechanism compared to other groups.

Conclusion: This study revealed an activation network similar to the emotional and inhibitory control networks observed in drug abusers in previous works. The results of DCM analysis also support the regulatory role of frontal regions on bottom regions. Furthermore, this study demonstrates the different effective connectivity patterns after drug abuse treatment and in this way helps the experts in the field.

* Corresponding Author:

Amir Homayoun Jafari, PhD

Address: Department of Nouroimaging and Analysis, Imam Khomeini Hospital Complex, Tehran University of Medical Sciences, Tehran, Iran Tel: +98 (912) 3504106

E-mail: h_jafari@sian.tums.ac.ir 


\section{Introduction}

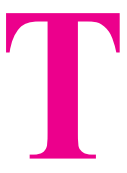

he neurocognitive process of drug craving in chronic drug abusers has been studied before and the brain regions involved in this process are well recognized (Wilson, Sayette, \& Fiez, 2004; Sutherland, McHugh, Pariyadath, \& Stein, 2012; Tang, Fellows, Small, \& Dagher, 2012; Yalachkov, Kaiser, Naumer, 2012). Previous studies have reported the key role of the amygdala and prefrontal cortex in the cue-induced craving process (Bechara, Damasio, Damasio, \& Lee, 1999). When exposed to drug cues, the brain regions, such as ventromedial prefrontal cortex (VMPFC) (Ben-Shahar, et al. 2013), dorsolateral prefrontal cortex (DLPFC) (Wilson et al. 2004; George and Koob 2013; Hayashi, Ko, Strafella, \& Dagher, 2013; Batista, Klauss, Fregni, Nitsche, \& Nakamura-Palacios, 2015), ventral striatum (Naqvi and Bechara 2009), and amygdala (Bechara, Damasio, \& Damasio, 2003) display activation in different drug dependents.

The associated brain regions do not act alone but work as parts of hidden networks. The recent studies have tried to find out and quantify these networks (Chase, Eickhoff, Laird, \& Hogarth 2011; Sutherland et al., 2013). The existing interactions between brain regions (nodes) can be passive or active; the passive type is called functional connectivity and the active one effective connectivity. Effective connectivity follows the theory of causality (Pearl, 2009). The causality in brain networks has been studied before, but the drug craving networks have been investigated in a few studies (Ray, Haney, Hanson, Biswal, \& Hanson, 2015). Based on some studies, chronic drug use can change the pattern of brain activation networks in drug dependents when exposing to drug cues (Goudriaan, de Ruiter, Van Den Brink, Oosterlaan, \& Veltman 2010; Janes, et al. 2010; Ma, et al, 2011; Lu, et al., 2012; Cisler, et al., 2013; Ding and Lee 2013a; Ding and Lee 2013b; Yang, et al., 2014). Furthermore, the regulatory effect of cortex on subcortical regions has already been proven, and their interactions follow a causal network pattern (Bechara, et al., 2001).

The causal networks can be quantified using different methods. Some methods address just the existence of the networks, but some other seek deeper to find more details. Two interesting issues in these networks are first how regions affect each other and second how they affect the relation among the regions. These networks can be quantified using effective connectivity measurement methods such as Structural Equation Modeling (SEM) (McLntosh \& Gonzalez-Lima 1994; Buchel, 1997; Astolfi, et al. 2004; Laird, et al., 2008), Granger causal- ity modeling (GCM) (Roebroeck, Formisano, Goebel, 2005; Wang, Chen, Bressler, \& Ding. 2007; Sato, et al., 2010), and dynamic causal modeling (DCM).

We hypothesized that the fronto-amygdalar regulation is complex and not only the prefrontal regions such as VMPFC and DLPFC have reciprocal modulatory effects on the amygdala, but also they have indirect causal effects. The differences in the effective connectivity networks were investigated between the following three groups in our study: one group included subjects with no history of drug dependence as the control group, one group included subjects who were successfully treated drug abuse with Methadone Maintenance Therapy (MMT), and the last group included subjects who were successfully treated drug abusers with Abstinence Based Therapy (ABT) method.

\section{Methods}

The Ethics Committee of Tehran University of Medical Sciences approved the study protocol and consent form. Before scanning, the imaging procedure was described for all subjects and their written informed consents were obtained. After scanning, a counseling procedure was done for each subject to check for any probable adverse effect on the subject's mental health, after presentation of drug-related cues.

\subsection{Participants}

Three study groups, each including 20 male subjects, were scanned. One group included subjects (with at least 3 months of opiate abstinence) who were successfully treated by MMT based method; the second group (with at least 3 months of opiate abstinence) included subjects who were successfully treated by ABT based method; and the third group comprised control subjects agematched with two other groups, who did not have any history of drug abuse. The demographic characteristics of the three groups are presented in Table 1.

\subsection{Functional magnetic resonance imaging task}

The task was a block design task containing 6 consecutive runs. Each run included one rest block of $24 \mathrm{~s}$ length (a cross was shown), one block of $24 \mathrm{~s}$ length as neutral (4 images not related to heroin, each for $6 \mathrm{~s}$, were shown to the subject), a second rest block, and a block of $24 \mathrm{~s}$ length as craving stimuli (4 images related to heroin, each for $6 \mathrm{~s}$, were shown to the subject). The images ( 24 heroin-related and 24 neutral) were selected from International Affective Picture System (Lang, Bradley, \& Cuthbert, 2005). The structure of the task is displayed in Figure 1. 


\subsection{Functional magnetic resonance imaging data acquisition}

Functional images were acquired with an Avanto 1.5T scanner (Siemens, Germany) with 8 channel head coil. The $\mathrm{T} 2 *$-weighted images were acquired with $\mathrm{TR}=3000 \mathrm{~ms}$, $\mathrm{TE}=50 \mathrm{~ms}$, flip angel $=90^{\circ}$, voxel size of $3 \times 3 \times 3 \mathrm{~mm}^{3}$, and matrix size of $64 \times 64$. Each volume was composed of 36 slices which covered the whole brain in axial direction. Structural image was acquired with the following specification: T1-weighted with $\mathrm{TR}=1910, \mathrm{TE}=3.55 \mathrm{~ms}$, flip angel $=30^{\circ}$, voxel size of $1 \times 1 \times 1 \mathrm{~mm}^{3}$, and matrix size of $256 \times 256$. The stimuli were presented using MR compatible goggles.

\subsection{Preprocessing}

FSL5 (Jenkinson, Beckmann, Behrens, Woolrich, Smith, 2012) MCFLIRT (Jenkinson \& Smith 2001; Jenkinson, Bannister, Brady, \& Smith, 2002) was used to correct the EPI images for the head motion. Slice timing correction was done using interleaved order, high-pass temporal filtering was done with the size of $96 \mathrm{~s}$ to remove the signal trend, a 3D Gaussian kernel with the size of $5 \mathrm{~mm}$ FWHM was used to smooth the functional images, and for group comparison the intensity normalization was done as the last part of the preprocessing step.

\subsection{Data analysis}

The purpose of this study was not to examine the between group differences with regard to regional activations, so we did only within group analyses. Using FLAME (FMRIB's Local Analysis of Mixed Effects), we included all 4 possible contrasts; i.e., craving, neutral, craving $>$ neutral, and neutral $>$ craving. Based on the results, only the craving>neutral contrast supports the idea of stronger activation during watching craving cues vs. watching neutral images.

\subsection{Time-series extraction}

According to our neuroscientific hypothesis, we chose 4 regions of interests (ROIs): VMPFC, DLPFC, ventral striatum, and amygdala. These regions have been shown to be active during a drug craving task. First we made a mask for each region in MNI space, then using transformation matrices, the masks were resliced and registered to each subject's EPI images. These matrices were calculated during registration in preprocessing step (standard2example_func.mat) and applied using the ApplyXFM tool in FSL5. The greatest eigenvariate of the voxels in each region was used as the time-series of the ROI. The extraction of eigenvariates from the time series across the voxels within each ROI was done using a singular value decomposition (SVD) method (Alter, Brown, Botstein, 2000). We used SPM12 ${ }^{1}$ Eigenvariate Tool for achieving this purpose.

\subsection{Dynamic causal modeling}

Effective connectivity means the causal interrelation of the regions in the brain; however, this relation is in the neuronal level which cannot be measured by fMRI. Dynamic causal modeling as an established method to quantify the effective connectivity includes 4 connectivity matrices which display the strength of interconnections. The first matrix (A) contains the strength of endogenous links; these are the interrelations of regions in the absence of any input, the second matrix (B) contains the strength related to the effects of inputs on the links between regions, the third matrix $(C)$ contains the direct strength of links of input effects on the regions and the last matrix (D) shows the strength of nonlinear links, which exerts from regions on the links connecting other regions. The equation which dominates the relation of these matrices is as follows:

$$
z=f(z, u, \theta)=A z+\left\{\sum_{j=1}^{m} u, B(j)+\sum_{i=1}^{n} z_{i} D(i)\right\} z+C u
$$

Computing DCM for a group of subjects include some steps, which are shown in the Figure 2. Our model space contained 38 models, which reciprocally connected 4 regions; the craving input emerged to various regions; linear and nonlinear links; and self-inhibitory links. The diverse models in the model space were used to answer different neuroscientific questions. Next we estimated all models for each subject to reach the exceedance probability measure for single subject analysis and these measures were used in the Bayesian model selection (BMS) (Stephan, Penny, Daunizeau, Moran, \& Friston, 2009) process to compare the models. Evidently, comparing single models does not simply provide any useful information, however, dividing the model space into families with similar features can yield the best result (Penny, et al., 2010). Thus, we divided the models into families according to their nonlinear links; separating linear and nonlinear models. Bayesian Model Averaging (BMA) was used to reach the final model. Also, we used SPM DCM12 Toolbox for computing the DCM network.

\section{Results}

\subsection{GLM results}

Statistical analysis of fMRI data of each group was done using FSL5 and the results indicated activations

1. www.fil.ion.ucl.ac.uk/spm 
in all regions of interest. Figure 3 depicts the activation patterns in one of the defined contrasts (craving>neutral) and Tables 2, 3, and 4 present the group level results for all study groups (same contrast).

\subsection{Dynamic causal modeling results}

The time-series of each region was extracted according to the method introduced in the previous section. The DCM estimation process was done for each model in the model space and the resulting exceedance probabilities were used in the process of BMS algorithm. Family par- titioning was done according to the nonlinear links and using BMA, the final DCM networks for all groups were calculated. The BMA results are presented in Table 5. Considering 4 connectivity matrices, this table is divided into 4 sections (highlighted with gray color).

The first section included the endogenous connections or the matrix A, the second section included the matrix B, the third section included matrix $\mathrm{C}$, and the last included matrix D. The first column of the table presents the start and the end of each connection (for connections which do not exist in all groups, there are no rows). The next 3 col-

Table 1. The demographic characteristics of the three study groups.

\begin{tabular}{|c|c|}
\hline ABT & Stats \\
\hline Age & $32 \pm 2.1$ \\
\hline Gender (male) & 15 \\
\hline Education (year) & $11.1 \pm 1.18$ \\
\hline Abstinence duration (month) & $15.6 \pm 4.1$ \\
\hline Opium abusers & 15 \\
\hline Heroin abusers & 15 \\
\hline Alcohol abusers & 12 \\
\hline Tobacco users & 15 \\
\hline \multicolumn{2}{|c|}{ MMT } \\
\hline Age & $34.7 \pm 2.52$ \\
\hline Gender (male) & 13 \\
\hline Education (year) & $11.2 \pm 1.7$ \\
\hline Abstinence duration (month) & $16.4 \pm 3.82$ \\
\hline Opium abusers & 13 \\
\hline Heroin abusers & 13 \\
\hline Alcohol abusers & 10 \\
\hline Tabaco users & 13 \\
\hline \multicolumn{2}{|c|}{ Control } \\
\hline Age & $28.9 \pm 2.55$ \\
\hline Gender (male) & 16 \\
\hline Education (year) & $13.2 \pm 1.46$ \\
\hline Alcohol abusers & 0 \\
\hline Tobacco users & 0 \\
\hline
\end{tabular}




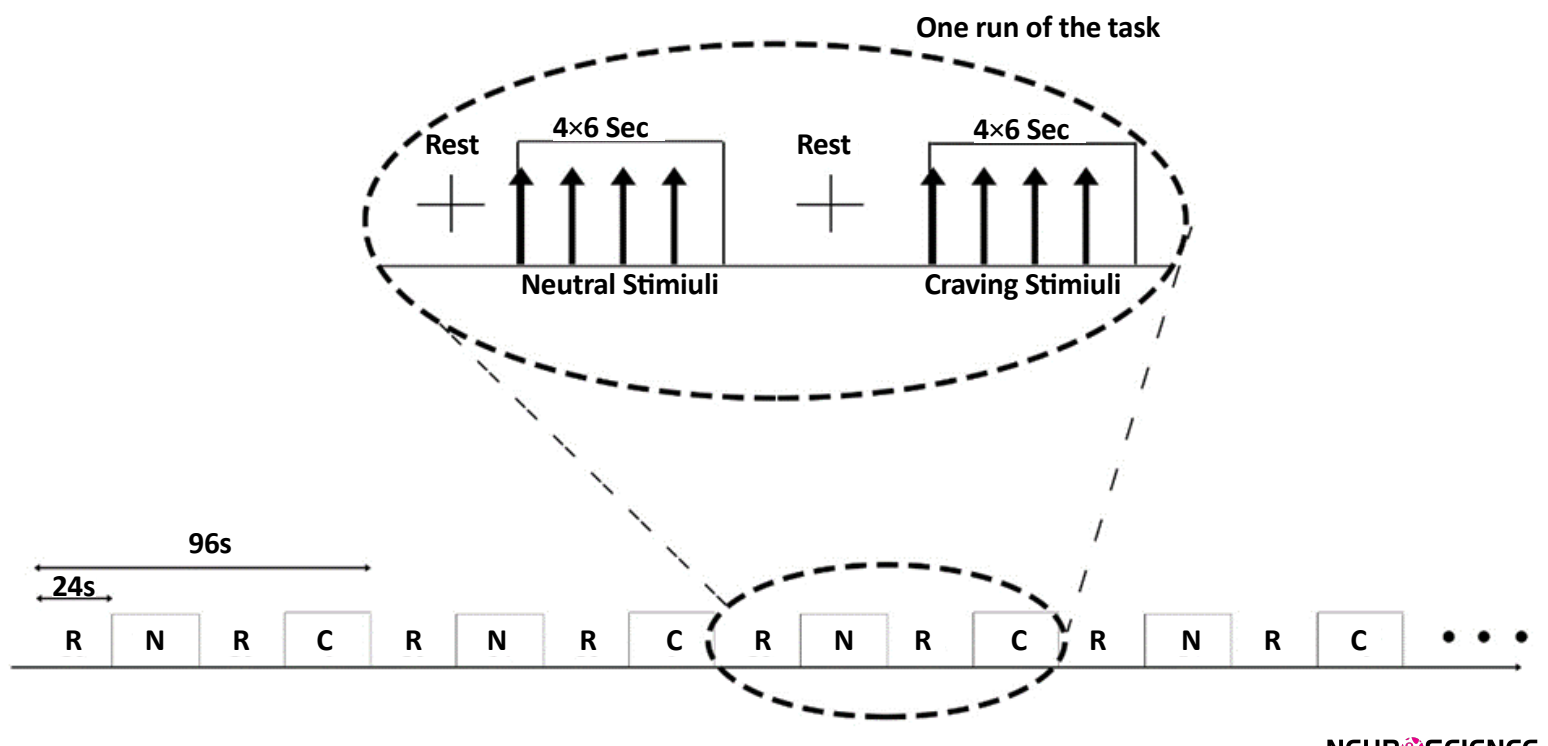

Figure 1. The task structure. R: represents Rest, C: represents Craving, and N: represents Neutral. There are 6 runs in the task each for 96 seconds.

umns are the mean strength of the named connection for each group. Zero number in the cells represents the lack of that connection in the relevant group. The numbers in these 3 columns represent different meanings with regard to effective connectivity theory; the change in the vari- ance of the links starting point will change the variance of the links ending point by the factor of the links mean strength. The sign of the number is directly related to the correlation of the variance change in two signals; positive means directly correlated and negative means correlation

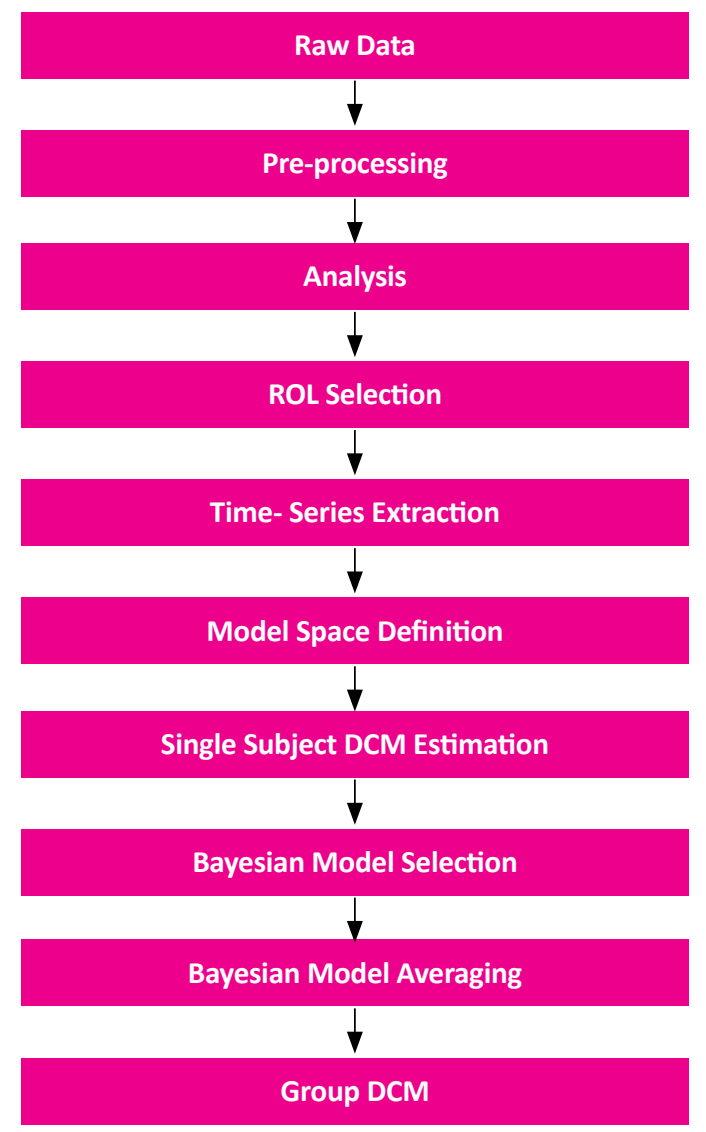

Figure 2. The diagram of calculating DCM. 
Table 2. The group level activation results in (Craving>Neutral) contrast for ABT group.

\begin{tabular}{|c|c|c|c|c|c|}
\hline \multirow{2}{*}{$\begin{array}{l}\text { Anatomical Regions } \\
\text { Supracalcarine cortex }\end{array}$} & \multirow[t]{2}{*}{ Cluster Size } & \multirow{2}{*}{$\begin{array}{c}\text { Z-values } \\
4.07\end{array}$} & \multicolumn{3}{|c|}{ Local Maximum Co-Ordinates } \\
\hline & & & 0 & -90 & 8 \\
\hline Lingual gyrus & & 3.96 & 2 & -84 & 6 \\
\hline \multirow[t]{2}{*}{ Lingual gyrus } & & 3.93 & -12 & -76 & -8 \\
\hline & 3123 & & & & \\
\hline Cuneus & & 3.91 & -10 & -98 & 12 \\
\hline Retrosplenial cortex & & 3.66 & 12 & -50 & 4 \\
\hline Cuneus & & 3.61 & 8 & -76 & 28 \\
\hline Superior parietal lobule & & 3.57 & -2 & -40 & 66 \\
\hline Primary somatosensory cortex & & 3.46 & 8 & -44 & 68 \\
\hline \multirow[t]{2}{*}{ Superior parietal lobule } & & 3.44 & -12 & -40 & 52 \\
\hline & 1228 & & & & \\
\hline Superior parietal lobule & & 3.41 & -18 & -42 & 42 \\
\hline Primary somatosensory cortex & & 3.29 & 0 & -26 & 62 \\
\hline Superior parietal lobule & & 3.28 & 14 & -48 & 72 \\
\hline Parahippocampal gyrus & & 3.73 & 26 & -44 & -8 \\
\hline \multirow[t]{2}{*}{ Fusiform cortex } & & 3.42 & 36 & -46 & -10 \\
\hline & 314 & & & & \\
\hline Parahippocampal gyrus & & 2.84 & 38 & -26 & -14 \\
\hline Fusiform cortex & & 2.58 & 38 & -30 & -16 \\
\hline Primary motor cortex & & 3.68 & 20 & -18 & 66 \\
\hline Primary motor cortex & & 3.52 & 32 & -28 & 62 \\
\hline Primary motor cortex & 293 & 3.46 & 36 & -30 & 66 \\
\hline Primary motor cortex & & 3.31 & 30 & -28 & 68 \\
\hline Primary motor cortex & & 2.75 & 12 & -28 & 72 \\
\hline \multirow[t]{2}{*}{ Parahippocampal gyrus } & & 3.51 & -20 & -42 & -10 \\
\hline & 258 & & & & \\
\hline Fusiform cortex & & 3.43 & -28 & -48 & -6 \\
\hline Heschl's gyrus & & 3.48 & 54 & -26 & 18 \\
\hline Secondary somatosensory cortex & & 3.36 & 52 & -28 & 24 \\
\hline Heschl's gyrus & & 2.85 & 62 & -24 & 12 \\
\hline & 226 & & & & \\
\hline Secondary somatosensory cortex & & 2.73 & 40 & -28 & 28 \\
\hline Supramarginal gyrus & & 2.61 & 48 & -38 & 14 \\
\hline Angular gyrus & & 2.46 & 58 & -36 & 18 \\
\hline
\end{tabular}




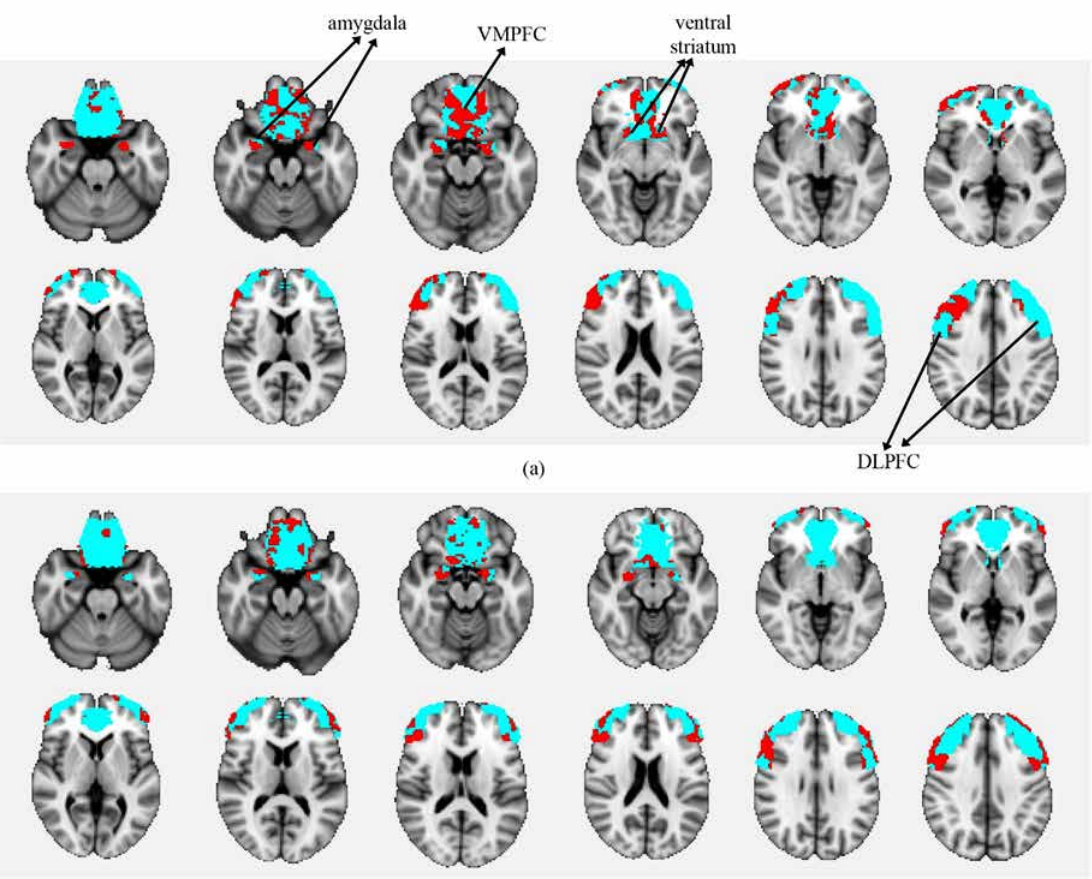

(b)
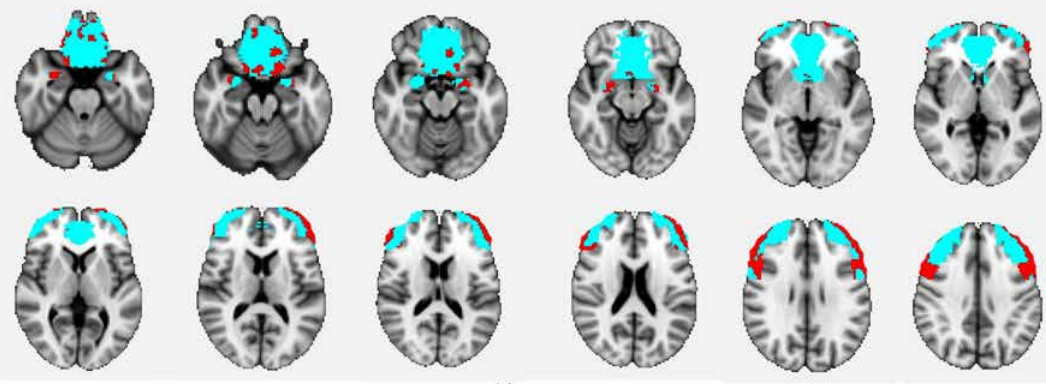

(c)

NEUROSCIENCE

Figure 3. Depicting the region-based activation analysis results for three study groups; (a) ABT, (b) MMT, and (c) Control. The blue regions are the ROIs in which the activations were investigated and the red regions are the active parts during the task and specifically the craving $>$ neutral contrast.

with phase lag of $180^{\circ}$. The last 3 columns of the table present significant statistically meaningful differences between the groups (top of each column) in that link (the first column). These networks are depicted in Figure 4.

With regard to the effective connectivity network for the control group, the craving input link only modulated the links between the regions, but there was no modulation for the other two groups and the input link only affected the amygdala. In the control group, DLPFC and amygdala affected ventral striatum but were not influenced by it. In the ABT group, VMPFC affected amygdala but there was no reverse effect. Finally in the MMT group, the relation from DLPFC to amygdala was a 1-way connection. Self-inhibitory connections in the control group were limited to VMPFC and ventral striatum regions. In the MMT group, only VMPFC had this self-inhibitory effect, while in the ABT group, all regions had the self-inhibitory effect. In the control group, DLPFC affected the connection of ventral striatum to VMPFC, and ventral striatum itself affected the connection of amygdala to DLPFC. In the ABT group, VMPFC influenced reciprocal connections between DLPFC and ventral striatum and also the connections between ventral striatum and amygdala. In the MMT group, the nonlinear link was from VMPFC to the link from DLPFC to ventral striatum.

\section{Discussion}

There are studies addressing the results of various treatment methods on drug abusers, but there is no conclusive evidence for superiority of any treatment method over others (Wang, et al., 2011). In addition, studies have shown 
Table 3. The group level activation results in (Craving>Neutral) contrast for MMT group.

\begin{tabular}{|c|c|c|c|c|c|}
\hline \multirow{2}{*}{$\begin{array}{c}\text { Anatomical Regions } \\
\text { Visual cortex }\end{array}$} & \multirow[t]{2}{*}{ Cluster Size } & \multirow{2}{*}{$\begin{array}{c}\text { Z-Values } \\
3.8\end{array}$} & \multicolumn{3}{|c|}{ Local Maximum Co-Ordinates } \\
\hline & & & 20 & -64 & 14 \\
\hline Lingual gyrus & & 3.6 & 0 & -80 & 22 \\
\hline Lingual gyrus & & 3.56 & 2 & -80 & 26 \\
\hline & 1426 & & & & \\
\hline Cuneus & & 3.45 & 22 & -64 & 2 \\
\hline Retrosplenial cortex & & 3.42 & -10 & -78 & 14 \\
\hline Cuneus & & 3.42 & -16 & -70 & 0 \\
\hline Insula & & 3.65 & -48 & -20 & 2 \\
\hline Inferior parietal lobule & & 3.57 & -60 & -38 & 20 \\
\hline Inferior parietal lobule & & 3.53 & -50 & -36 & 14 \\
\hline & 718 & & & & \\
\hline Inferior parietal lobule & & 3.43 & -54 & -34 & 12 \\
\hline Inferior parietal lobule & & 3.23 & -60 & -36 & 10 \\
\hline Insula & & 3.17 & -38 & -30 & 8 \\
\hline Primary somatosensory cortex & & 3.43 & 16 & -46 & 56 \\
\hline Primary somatosensory cortex & & 3.4 & 18 & -46 & 60 \\
\hline Superior parietal lobule & & 3.23 & 16 & -46 & 52 \\
\hline & 454 & & & & \\
\hline Superior parietal lobule & & 3.23 & 24 & -46 & 68 \\
\hline Superior parietal lobule & & 3.13 & 12 & -50 & 68 \\
\hline Postcentral gyrus & & 3.06 & 12 & -46 & 68 \\
\hline Secondary somatosensory cortex & & 3.44 & 50 & -10 & 24 \\
\hline Secondary somatosensory cortex & & 3.25 & 58 & -2 & 12 \\
\hline Primary somatosensory cortex & & 3.21 & 56 & -6 & 22 \\
\hline & 263 & & & & \\
\hline Secondary somatosensory cortex & & 3.08 & 60 & 0 & 4 \\
\hline Secondary somatosensory cortex & & 2.87 & 58 & -12 & 12 \\
\hline Secondary somatosensory cortex & & 2.85 & 64 & -14 & 10 \\
\hline Heschl's gyrus & & 3.3 & 56 & -28 & 14 \\
\hline Inferior parietal lobule & & 3.19 & 56 & -38 & 12 \\
\hline Inferior parietal lobule & & 3.18 & 58 & -48 & 10 \\
\hline & 262 & & & & \\
\hline Heschl's gyrus & & 3.12 & 60 & -28 & 16 \\
\hline Superior temporal gyrus & & 2.96 & 60 & -36 & 6 \\
\hline Inferior parietal lobule & & 2.91 & 46 & -42 & 12 \\
\hline
\end{tabular}


Table 4. The group level activation results in (Craving>Neutral) contrast for the control group.

\begin{tabular}{|c|c|c|c|c|c|}
\hline \multirow{2}{*}{$\begin{array}{c}\text { Anatomical Regions } \\
\text { Visual cortex }\end{array}$} & \multirow[t]{2}{*}{ Cluster Size } & \multirow{2}{*}{$\begin{array}{c}\text { Z-Values } \\
3.97\end{array}$} & \multicolumn{3}{|c|}{ Local Maximum Co-Ordinates } \\
\hline & & & -12 & -58 & 0 \\
\hline Cuneus & \multirow{5}{*}{2214} & 3.94 & -12 & -46 & -6 \\
\hline Visual cortex & & 3.87 & -20 & -58 & 2 \\
\hline Visual cortex & & 3.82 & 16 & -62 & 8 \\
\hline Lingual gyrus & & 3.78 & 6 & -64 & 2 \\
\hline Visual cortex & & 3.69 & -8 & -92 & 8 \\
\hline Inferior parietal lobule & \multirow{6}{*}{705} & 4.19 & -46 & -34 & 26 \\
\hline Insula & & 3.51 & -30 & -36 & 12 \\
\hline Secondary somatosensory cortex & & 3.5 & -40 & -30 & 26 \\
\hline Insula & & 3.35 & -34 & -32 & 14 \\
\hline Insula & & 3.26 & -48 & -12 & 22 \\
\hline Primary somatosensory cortex & & 3.23 & -16 & -34 & 50 \\
\hline Supramarginal gyrus & \multirow{6}{*}{649} & 3.98 & 50 & -38 & 10 \\
\hline Middle temporal gyrus & & 3.51 & 54 & -42 & 2 \\
\hline Inferior parietal lobule & & 3.45 & 58 & -36 & 12 \\
\hline Insula & & 3.37 & 40 & -24 & 10 \\
\hline Inferior parietal lobule & & 3.35 & 62 & -32 & 18 \\
\hline Middle Temporal gyrus & & 3.24 & 64 & -36 & 2 \\
\hline Cingulate gyrus & \multirow{3}{*}{275} & 3.19 & -10 & -4 & 36 \\
\hline Primary motor cortex & & 2.71 & -32 & -16 & 36 \\
\hline Primary motor cortex & & 2.58 & -26 & -16 & 46 \\
\hline Inferior parietal lobule & \multirow{4}{*}{230} & 3.82 & -64 & -44 & 14 \\
\hline Inferior parietal lobule & & 3.56 & -48 & -42 & 10 \\
\hline Inferior parietal lobule & & 3.09 & -54 & -46 & 22 \\
\hline Inferior parietal lobule & & 2.81 & -62 & -36 & 12 \\
\hline
\end{tabular}

NEUR SIENCE

different brain activation patterns for treated subjects, however, currently there is no direct study of brain effective connectivity differences between variously treated subjects. The effective connectivity estimation can measure the regulatory effects of regions and craving cues on the different parts of the brain network and helps in better understanding of the craving mechanism (Kober, et al., 2010).

We investigated the effective connectivity network in 3 groups. Two groups included successfully treated drug abusers, with different treatment methods; one with MMT and the other with ABT. The third group included individuals with no history of drug abuse. The results of the last group helped us provide a basic network for brain effective connectivity pattern, when no prior bias exist. The networks were estimated between 4 regions of interest which were mentioned in previous studies.
Studies have indicated that cue exposure increases craving and results in more activities in these regions. These regions are also associated with emotion.

The implication of prefrontal regions in cognitive control (Wrase, et al., 2007; de Greck, et al., 2009) and its regulatory effect on emotion specific regions like amygdala and ventral striatum have been already proven (Franklin, et al., 2007; Meda et al., 2009). The resulted networks showed the active role of these regions in the network and by using DCM, the difference of the networks between these 3 groups were depicted numerically and structurally.

\subsection{The craving input effect}

The differences between the control group and two treated groups indicated that the cue-induced pictures did 


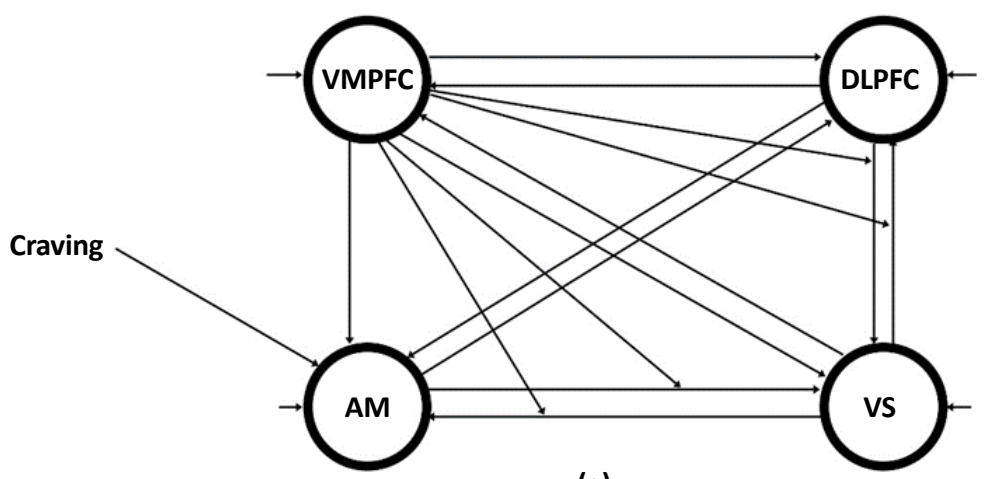

(a)

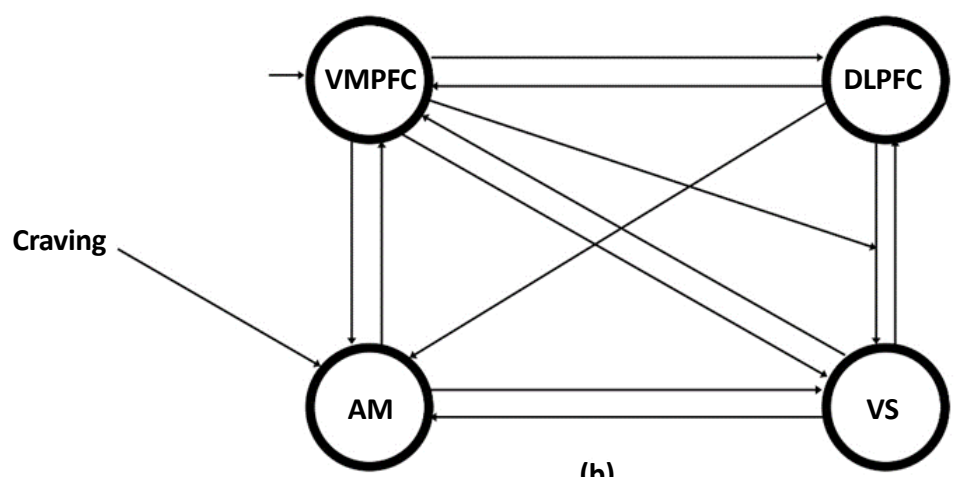

(b)

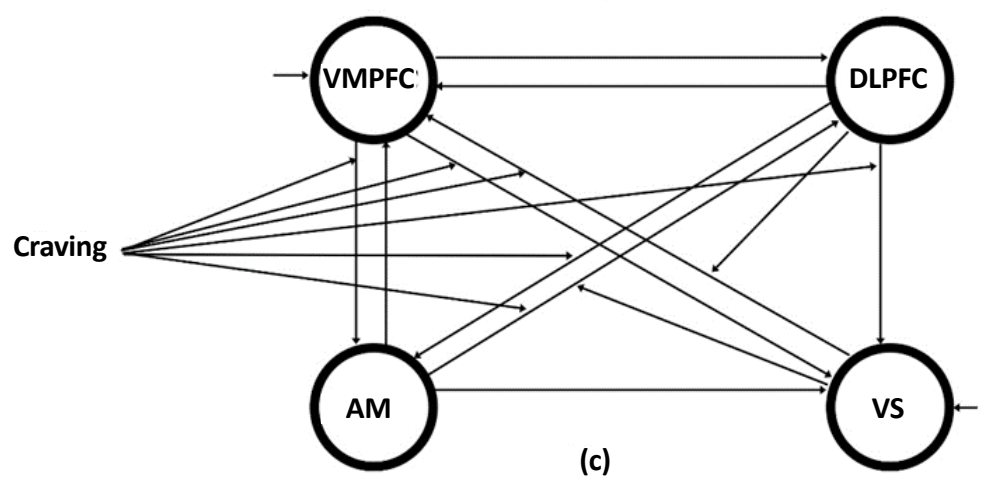

NEUR:SCIENCE

Figure 4. The DCM network structure for three groups; (a) the final network for ABT group, (b) The final network for MMT group, and (c) The final network for the control group.

not affect the emotion of healthy subjects, but affected amygdala, causing emotional indices in the other groups. To the healthy subjects, these pictures seem as neutral pictures and only modulate some of the links in the network (as perturbations for the task). This finding is in accordance with previous studies which mentioned the role of amygdala in cue-induced tasks in subjects with a history of drug abuse (Bechara, et al., 1999; Bechara, et al., 2003; Goudriaan, et al., 2010; Chase, et al., 2011; Tang et al., 2012; Ray, et al., 2015).
4.2. The modulatory effect of ventromedial prefrontal cortex

Studies have already proven the role of prefrontal-striatal pathway in the control of craving to drug use (Koob, 2001; Volkow, Fowler, Wang, 2003; Everitt \& Robbins, 2005). The lack of control over drug taking is considered a sign of addiction and is critical in relapse. Frontal brain regions have an important role in inhibitory control of this behavior. It has been shown that VMPFC has modulatory effects on other regions, including amygdale and ventral striatum in the cue-induced craving tasks (Becha- 
Table 5. The BMA results. The links with zero strength in the table were not significant in their own groups. The last three columns of the table compare between groups with the P-values used in the test. In these columns "ns" means not significant.

\begin{tabular}{|c|c|c|c|c|c|c|}
\hline & Control & ABT & MMT & \multicolumn{3}{|c|}{ Significance } \\
\hline & Mean Strength & Mean Strength & Mean Strength & $\begin{array}{c}\text { Control- } \\
\text { ABT }\end{array}$ & $\begin{array}{c}\text { Control- } \\
\text { MMT }\end{array}$ & АВT-MMT \\
\hline \multicolumn{7}{|c|}{ Endogenous Connections } \\
\hline VMPFC to VMPFC & -0.1 & -0.2 & -0.1 & ns & ns & ns \\
\hline DLPFC to DLPFC & 0 & -0.8 & 0 & $P<0.01$ & ns & $P<0.01$ \\
\hline VS to VS & -0.1 & -0.1 & 0 & ns & $P<0.05$ & $P<0.05$ \\
\hline$A M$ to $A M$ & 0 & -0.1 & 0 & $\mathrm{P}<0.05$ & ns & $P<0.05$ \\
\hline VMPFC to DLPFC & 0.2 & 0.2 & 0.1 & ns & ns & ns \\
\hline DLPFC to VMPFC & 0.2 & 0.8 & 0.1 & $\mathrm{P}<0.01$ & ns & $\mathrm{P}<0.01$ \\
\hline VMPFC to VS & 0.4 & 0.3 & 0.2 & $\mathrm{P}<0.05$ & $P<0.05$ & ns \\
\hline VS to VMPFC & 0.1 & 0.1 & 0.1 & ns & ns & ns \\
\hline VMPFC to AM & 0.3 & 0.5 & 0.2 & $P<0.05$ & ns & $\mathrm{P}<0.05$ \\
\hline AM to VMPFC & 0.2 & 0 & 0.2 & $\mathrm{P}<0.05$ & ns & $P<0.05$ \\
\hline DLPFC to VS & 0.3 & -0.4 & 0.1 & $P<0.01$ & $P<0.01$ & $P<0.01$ \\
\hline VS to DLPFC & 0 & 0.2 & 0.1 & $\mathrm{P}<0.05$ & $P<0.05$ & ns \\
\hline DLPFC to AM & 0.1 & 0.6 & 0.1 & $P<0.01$ & ns & $P<0.01$ \\
\hline AM to DLPFC & 0.1 & -0.2 & 0 & $P<0.01$ & $P<0.05$ & $P<0.01$ \\
\hline VS to AM & 0 & 0.2 & 0.1 & $P<0.05$ & $P<0.05$ & ns \\
\hline AM to VS & 0.2 & 0.2 & 0.3 & ns & ns & ns \\
\hline \multicolumn{7}{|c|}{ Craving Input } \\
\hline AM & 0 & 0.7 & 0.1 & $P<0.01$ & $\mathrm{P}<0.05$ & $\mathrm{P}<0.05$ \\
\hline \multicolumn{7}{|c|}{ Craving Modulation } \\
\hline VMPFC to VS & 0.1 & 0 & 0 & $P<0.05$ & $\mathrm{P}<0.05$ & ns \\
\hline VS to VMPFC & 0.1 & 0 & 0 & $P<0.05$ & $P<0.05$ & ns \\
\hline VMPFC to AM & 0.2 & 0 & 0 & $\mathrm{P}<0.05$ & $P<0.05$ & ns \\
\hline DLPFC to VS & -0.3 & 0 & 0 & $P<0.05$ & $P<0.05$ & ns \\
\hline DLPFC to AM & 0.1 & 0 & 0 & $P<0.05$ & $P<0.05$ & ns \\
\hline AM to DLPFC & 0.1 & 0 & 0 & $P<0.05$ & $P<0.05$ & ns \\
\hline \multicolumn{7}{|c|}{ Nonlinear Connections } \\
\hline VMPFC to DLPFC-VS & 0 & -0.1 & 0.1 & $P<0.05$ & ns & $P<0.01$ \\
\hline VMPFC to VS-DLPFC & 0 & 0.4 & 0 & $\mathrm{P}<0.01$ & ns & $\mathrm{P}<0.01$ \\
\hline VMPFC to VS-AM & 0 & 0.2 & 0 & ns & ns & ns \\
\hline VMPFC to AM-VS & 0 & -0.2 & 0 & $\mathrm{P}<0.01$ & ns & $\mathrm{P}<0.01$ \\
\hline DLPFC to VS-VMPFC & -0.2 & 0 & 0 & $P<0.01$ & $P<0.01$ & ns \\
\hline VS to AM-DLPFC & -0.2 & 0 & 0 & $P<0.01$ & $P<0.01$ & ns \\
\hline
\end{tabular}


ra, et al., 1999; Bechara 2005; Lu, et al., 2012; Sladky, et al., 2013). In our study by considering the modulatory effect of VMPFC on other interregional connections, it was revealed that in healthy subjects there was no modulatory effects, as they did not have any emotional responses to craving specific pictures.

However, the VMPFC modulatory effects in ABT group indicate the important role of this region in drug resistance among these subjects. This group are trained to resist drug taking and the results reveal that the VMPFC acts as a part of top neural system, which tries to control the emotional decisions. This finding is in accordance with the findings of prior studies on the regulation of negative emotions (Ochsner \& Gross, 2008) and positive emotions (Kim and Hamann, 2007; Delgado, Gillis, Phelps, 2008). They reported the activation of these prefrontal systems and suggested the role of cognition to regulate responses to affective cues; for example drug cues. In the MMT group, we did not expect to observe any modulatory effect as the subjects took a dose of methadone and they would seem to have normal behavior when encountering cueinduced images. The only modulatory effect of VMPFC in this group was observed in the DLPFC-ventral striatum link, which was not statistically different from the control group who did not have this link (in the control group this link was not statistically significant).

The importance of DLPFC as a part of prefrontal cortical area has been highlighted in previous addictive-cue studies (Goldstein \& Volkow, 2002; Wilson et al., 2004; Goldstein, et al., 2007; Volkow, Fowler, Wang, Baler, \& Telang, 2009). The neural activity of DLPFC may be modulated by sensory information, motivational state, and task contingencies (Miller, 2000). In our study, DLPFC exhibited an important role in regulatory processing of cognitive and motivationally relevant information.

In this study, we identified 3 effective connectivity networks for 3 different groups, with various structures and links strength. These results can prove the different effects of treatment methods and their underlying neuronal mechanisms. The vast modulatory effect of VMPFC occur only in ABT group. This effect can prove the inhibitory role of this brain region in drug craving and also indicate the successful treatment of the subjects in this group, as the main target of this treatment is to train the patients to control their craving. The modulatory effect of VMPFC in MMT group, in contrast with the other treatment group, is a single effect which can exist because of the anatomical connections between these regions.
In the control group, the input data did not directly affect the regions and only modulates the links. In this network, input is on when the craving images are shown and is off in other situations. Thus, the input can be interpreted as watching craving-related images and this exclusive modulatory effect can be the result of subjects' inattentiveness to these cues. These results cannot prove the superiority of one method over another but at least may help choose the best method for different subjects in various situations.

\section{Acknowledgements}

The present paper was extracted from the $\mathrm{PhD}$ thesis of the first author in the Department of Medical Physics and Biomedical Engineering, School of Medicine, Tehran University of Medical Sciences.

\section{Conflict of Interest}

The authors declared no conflicts of interest.

\section{References}

Alter, O., Brown, P. O., \& Botstein, D. (2000). Singular value decomposition for genome-wide expression data processing and modeling. Proceedings of the National Academy of Sciences, 97(18), 10101-6. doi: 10.1073/pnas.97.18.10101

Astolfi, L., Cincotti, F., Mattia, D., Salinari, S., Babiloni, C., Basilisco, A., et al. (2004). Estimation of the effective and functional human cortical connectivity with structural equation modeling and directed transfer function applied to high-resolution EEG. Magnetic Resonance Imaging, 22(10), 1457-70. doi: 10.1016/j.mri.2004.10.006

Batista, E. K., Klauss, J., Fregni, F., Nitsche, M. A., \& NakamuraPalacios, E. M. (2015). A randomized placebo-controlled trial of targeted prefrontal cortex modulation with bilateral tdcs in patients with crack-cocaine dependence. International Journal of Neuropsychopharmacology, 18(12), 66. doi: 10.1093/ijnp/ pyv066

Bechara, A. (2005). Decision making, impulse control and loss of willpower to resist drugs: A neurocognitive perspective. $\mathrm{Na}$ ture Neuroscience, 8(11), 1458-63. doi: 10.1038/nn1584

Bechara, A., Damasio, H., \& Damasio, A. R. (2003). Role of the amygdala in decision-making. Annals of the New York Academy of Sciences, 985(1), 356-9. doi: 10.1111/j.1749-6632.2003. tb07094.x

Bechara, A., Damasio, H., Damasio, A. R. \& Lee, G. P. (1999) Different contributions of the human amygdala and ventromedial prefrontal cortex to decision-making. Journal of Neuroscience 19(13), 5473-5481. PMID: 10377356 
Bechara, A., Dolan, S., Denburg, N., Hindes, A., Anderson, S. W., \& Nathan P. E. (2001). Decision-making deficits, linked to a dysfunctional ventromedial prefrontal cortex, revealed in alcohol and stimulant abusers. Neuropsychologia, 39(4), 376-89. PMID: 11164876

Ben-Shahar, O., Sacramento, A. D., Miller, B. W., Webb, S. M., Wroten, M. G., Silva, H. E., et al. (2013). Deficits in ventromedial prefrontal cortex group 1 metabotropic glutamate receptor function mediate resistance to extinction during protracted withdrawal from an extensive history of cocaine self-administration. Journal of Neuroscience, 33(2), 495-506. doi: 10.1523/ jneurosci.3710-12.2013

Buchel, C. (1997). Modulation of connectivity in visual pathways by attention: cortical interactions evaluated with structural equation modelling and fMRI. Cerebral Cortex, 7(8), 768-78. doi: $10.1093 /$ cercor/7.8.768

Chase, H. W., Eickhoff, S. B., Laird, A. R., \& Hogarth, L. (2011). The neural basis of drug stimulus processing and craving: An activation likelihood estimation meta-analysis. Biological Psychiatry, 70(8), 785-93. doi: 10.1016/j.biopsych.2011.05.025

Cisler, J. M., Elton, A., Kennedy, A. P., Young, J., Smitherman, S., Andrew James, G., et al. (2013). Altered functional connectivity of the insular cortex across prefrontal networks in cocaine addiction. Psychiatry Research: Neuroimaging, 213(1), 39-46. doi: 10.1016/j.pscychresns.2013.02.007

de Greck, M., Supady, A., Thiemann, R., Tempelmann, C., Bogerts, B., Forschner, L., et al. (2009). Decreased neural activity in reward circuitry during personal reference in abstinent alcoholics-A fMRI study. Human Brain Mapping, 30(5), 16911704. doi: 10.1002/hbm.20634

Delgado, M. R., Gillis, M. M., \& Phelps, E. A. (2008). Regulating the expectation of reward via cognitive strategies. Nature Neuroscience, 11(8), 880-1. doi: 10.1038/nn.2141

Ding, X., \& Lee, S. W. (2013a). Changes of functional and effective connectivity in smoking replenishment on deprived heavy smokers: a resting-state fMRI study. PLOS ONE, 8(3), 59331. doi: 10.1371/journal.pone.0059331

Ding, X., \& Lee, S. W. (2013b). Cocaine addiction related reproducible brain regions of abnormal default-mode network functional connectivity: A group ICA study with different model orders. Neuroscience Letters, 548, 110-4. doi: 10.1016/j. neulet.2013.05.029

Everitt, B. J., \& Robbins, T. W. (2005). Neural systems of reinforcement for drug addiction: from actions to habits to compulsion. Nature Neuroscience, 8(11), 1481-9. doi: 10.1038/ nn1579

Franklin, T. R., wang, Z., Wang, J., Sciortino, N., Harper, D., $\mathrm{Li}$, Y., et al. (2007). Limbic activation to cigarette smoking cues independent of nicotine withdrawal: A perfusion fMRI study. Neuropsychopharmacology, 32(11), 2301-9. doi: 10.1038/ sj.npp.1301371

George, O., \& Koob, G. F. (2013). Control of craving by the prefrontal cortex. Proceedings of the National Academy of Sciences, 110(11), 4165-6. doi: 10.1073/pnas.1301245110

Goldstein, R. Z., Tomasi, D., Rajaram, S., Cottone, L. A., Zhang, L., Maloney, T., et al. (2007). Role of the anterior cingulate and medial orbitofrontal cortex in processing drug cues in cocaine addiction. Neuroscience 144(4): 1153-9.
Goldstein, R. Z., \& Volkow, N. D. (2002). Drug Addiction and Its Underlying Neurobiological Basis: Neuroimaging Evidence for the Involvement of the Frontal Cortex. American Journal of Psychiatry, 159(10), 1642-52. doi: 10.1176/appi.ajp.159.10.1642

Goudriaan, A. E., De Ruiter, M. B., Van Den Brink, W., Oosterlaan, J., \& Veltman, D. J. (2010). Brain activation patterns associated with cue reactivity and craving in abstinent problem gamblers, heavy smokers and healthy controls: an fMRI study. Addiction Biology, 15(4), 491-503. doi: 10.1111/j.13691600.2010.00242.x

Hayashi, T., Ko, J. H., Strafella, A. P., \& Dagher, A. (2013) Dorsolateral prefrontal and orbitofrontal cortex interactions during self-control of cigarette craving. Proceedings of the National Academy of Sciences, 110(11), 4422-7. doi: 10.1073/ pnas. 1212185110

Janes, A. C., Pizzagalli, D. A., Richardt, S., Frederick, B. deB., Chuzi, S., Pachas, G., et al. (2010). Brain reactivity to smoking cues prior to smoking cessation predicts ability to maintain tobacco abstinence. Biological Psychiatry, 67(8), 722-9. doi: 10.1016/j.biopsych.2009.12.034

Jenkinson, M., Bannister, P., Brady, M., \& Smith, S. (2002). Improved optimization for the robust and accurate linear reg istration and motion correction of brain images. NeuroImage, 17(2), 825-41. doi: 10.1006/nimg.2002.1132

Jenkinson, M., Beckmann, C. F., Behrens, T. E. J., Woolrich, M. W., \& Smith, S. M. (2012). FSL. NeuroImage, 62(2), 782-790. doi: 10.1016/j.neuroimage.2011.09.015

Jenkinson, M., \& Smith, S. (2001). A global optimisation method for robust affine registration of brain images. Medical Image Analysis, 5(2), 143-56. doi: 10.1016/s1361-8415(01)00036-6

Kim, S. H., \& Hamann, S. (2007). Neural correlates of positive and negative emotion regulation. Journal of Cognitive Neuroscience, 19(5), 776-98. doi: 10.1162/jocn.2007.19.5.776

Kober, H., Mende-Siedlecki, P., Kross, E. F., Weber, J., Mischel, W., Hart, C. L., et al. (2010). Prefrontal-striatal pathway underlies cognitive regulation of craving. Proceedings of the $\mathrm{Na}$ tional Academy of Sciences, 107(33), 14811-16. doi: 10.1073/ pnas. 1007779107

Koob, G. (2001). Drug addiction, dysregulation of reward, and allostasis. Neuropsychopharmacology, 24(2), 97-129. doi: 10.1016/s0893-133x(00)00195-0

Laird, A. R., Robbins, J. M., Li, K., Price, L. R., Cykowski, M. D. Narayana, S., et al. (2008). Modeling motor connectivity using TMS/PET and structural equation modeling. Neurolmage, 41(2), 424-36. doi: 10.1016/j.neuroimage.2008.01.065

Lang, P. J., Bradley, M. M., \& Cuthbert,B. N. (2005). International Affective Picture System (IAPS): Affective ratings of pictures and instruction manual. Gainesville: Center for the Study of Emotion \& Attention.

Lu, Q., Li, H., Luo, G., Wang, Y., Tang, H., Han, L., \& Yao, Z (2012). Impaired prefrontal-amygdala effective connectivity is responsible for the dysfunction of emotion process in major depressive disorder: A dynamic causal modeling study on MEG. Neuroscience Letters, 523(2), 125-30. doi: 10.1016/j.neulet.2012.06.058

Ma, N., Liu, Y., Fu, X. M., Li, N., Wang, C. X., Zhang, H., et al. (2011). Abnormal brain default-mode network functional con- 
nectivity in drug addicts. PLoS ONE, 6(1), 16560. doi: 10.1371/ journal.pone. 0016560

McLntosh, A. R., \& Gonzalez-Lima, F. (1994). Structural equation modeling and its application to network analysis in functional brain imaging. Human Brain Mapping, 2(1-2), 2-22. doi: 10.1002/hbm.460020104

Meda, S. A., Calhoun, V. D., Astur, R. S., Turner, B. M., Ruopp, K., \& Pearlson, G. D. (2009). Alcohol dose effects on brain circuits during simulated driving: An fMRI study. Human Brain Mapping, 30(4), 1257-70. doi: 10.1002/hbm.20591

Miller, E. K. (2000). The prefrontal cortex and cognitive control. Nature Reviews Neuroscience, 1(1), 59-65. doi: 10.1038/35036228

Naqvi, N. H., \& Bechara, A. (2009). The hidden island of addiction: the insula. Trends in Neurosciences, 32(1), 56-67. doi: 10.1016/j.tins.2008.09.009

Ochsner, K. N., \& Gross, J. J. (2008). Cognitive Emotion Regulation. Current Directions in Psychological Science, 17(2), 153-8. doi: 10.1111/j.1467-8721.2008.00566.x

Pearl, J. (2009). Causality: Models, reasoning and inference. Cambridge: Cambridge University Press.

Penny, W. D., Stephan, K. E., Daunizeau, J., Rosa, M. J., Friston, K. J., Schofield, T. M., et al. (2010). Comparing Families of Dynamic Causal Models. PLoS Computational Biology, 6(3), 1000709. doi:10.1371/journal.pcbi.1000709

Ray, S., Haney, M., Hanson, C., Biswal, B., \& Hanson, S. J. (2015) Modeling causal relationship between brain regions within the drug-cue processing network in chronic cocaine smokers. Neuropsychopharmacology, 40(13), 2960-8. doi: 10.1038/ npp. 2015.150

Roebroeck, A., Formisano, E., \& Goebel, R. (2005). Mapping directed influence over the brain using Granger causality and fMRI. NeuroImage, 25(1), 230-42. doi: 10.1016/j.neuroimage.2004.11.017

Sato, J. R., Fujita, A., Cardoso, E. F., Thomaz, C. E., Brammer, M. J., \& Amaro, E. (2010). Analyzing the connectivity between regions of interest: An approach based on cluster Granger causality for fMRI data analysis. NeuroImage, 52(4), 1444-55. doi 10.1016/j.neuroimage.2010.05.022

Sladky, R., Höflich, A., Küblböck, M., Kraus, C., Baldinger, P., Moser, E., et al. (2013). Disrupted effective connectivity between the amygdala and orbitofrontal cortex in social anxiety disorder during emotion discrimination revealed by dynamic causal modeling for fMRI. Cerebral Cortex, 25(4), 895-903. doi: $10.1093 /$ cercor/bht279

Stephan, K. E., Penny, W. D., Daunizeau, J., Moran, R. J., \& Friston, K. J. (2009). Bayesian model selection for group studies. NeuroImage, 46(4), 1004-17. doi: 10.1016/j.neuroimage.2009.03.025

Sutherland, M. T., Carroll, A. J., Salmeron, B. J., Ross, T. J., Hong, L. E., \& Stein, E. A. (2013). Down-regulation of amygdala and insula functional circuits by varenicline and nicotine in abstinent cigarette smokers. Biological Psychiatry, 74(7), 538-46. doi:10.1016/j.biopsych.2013.01.03.

Sutherland, M. T., McHugh, M. J., Pariyadath, V., \& Stein, E. A. (2012). Resting state functional connectivity in addiction: Lessons learned and a road ahead. NeuroImage, 62(4), 2281-95. doi: 10.1016/j.neuroimage.2012.01.117
Tang, D. W., Fellows, L. K., Small, D. M., \& Dagher, A. (2012) Food and drug cues activate similar brain regions: A metaanalysis of functional MRI studies. Physiology \& Behavior, 106(3), 317-24. doi: 10.1016/j.physbeh.2012.03.009

Volkow, N. D., Fowler, J. S., \& Wang, G.-J. (2003). The addicted human brain: Insights from imaging studies. Journal of Clinical Investigation, 111(10), 1444-51. doi: 10.1172/jci200318533

Volkow, N. D., Fowler, J. S., Wang, G. J., Baler, R., \& Telang, F. (2009). Imaging dopamine's role in drug abuse and addiction. Neuropharmacology 56(Suppl 1), 3-8. 10.1016/j.neuropharm.2008.05.022

Wang, W., Q. Li, Y. Wang, J. Tian, W. Yang, W. Li, W. et al (2011). Brain fMRI and craving response to heroin-related cues in patients on methadone maintenance treatment. American Journal of Drug and Alcohol Abuse, 37(2), 123-30. doi: 10.3109/00952990.2010.543997

Wang, X., Chen, Y., Bressler, S. L., \& Ding, M. (2007). Granger causality between multiple interdependent neurobiological time series: blockwise versus pairwise methods. International Journal of Neural Systems, 17(2), 71-8. doi: 10.1142/ s0129065707000944

Wilson, S. J., Sayette, M. A., \& Fiez, J. A. (2004). Prefrontal responses to drug cues: a neurocognitive analysis. Nature Neuroscience, 7(3), 211-4. doi: 10.1038/nn1200

Wrase, J., Schlagenhauf, F., Kienast, T., Wüstenberg, T., Bermpohl, F., Kahnt, T., et al. (2007). Dysfunction of reward processing correlates with alcohol craving in detoxified alcoholics. NeuroImage, 35(2), 787-94. doi: 10.1016/j.neuroimage.2006.11.043

Yalachkov, Y., Kaiser, J., \& Naumer, M. J. (2012). Functiona neuroimaging studies in addiction: Multisensory drug stimuli and neural cue reactivity. Neuroscience \& Biobehavioral Reviews, 36(2), 825-35. doi: 10.1016/j.neubiorev.2011.12.004

Yang, W., Wang, F., Zhang, Z., Ren, X., Zhang, Z., Li, Y. \& Sun,T. (2014). [Altered effective connectivity of insula in nicotine addiction (Chinese)]. Journal of the Chinese Medical Association 94(21): 1667-70. PMID: 25152295 